\title{
Cats protecting birds revisited
}

\author{
Meng Fan ${ }^{\mathrm{a}}$, Yang Kuang ${ }^{\mathrm{b}, *}$, Zhilan Feng ${ }^{\mathrm{c}}$ \\ ${ }^{a}$ School of Mathematics and Statistics and Key Laboratory for Vegetation Ecology of the Education Ministry, \\ Northeast Normal University, 5268 Renmin Street, Changchun, Jilin 130024, People's Republic of China \\ ${ }^{\mathrm{b}}$ Department of Mathematics and Statistics, Arizona State University, Tempe, AZ 85287-1804, United States \\ ${ }^{\mathrm{c}}$ Department of Mathematics, Purdue University, West Lafayette, IN 47907-1395, United States
}

Received 18 October 2004; accepted 22 December 2004

\begin{abstract}
In this paper, we revisit the dynamical interaction among prey (bird), mesopredator (rat), and superpredator (cat) discussed in [Courchamp, F., Langlais, M., Sugihara, G., 1999. Cats protecting birds: modelling the mesopredator release effect. Journal of Animal Ecology 68, 282-292]. First, we develop a prey-mesopredator-superpredator (i.e., bird-rat-cat, briefly, BRC) model, where the predator's functional responses are derived based on the classical Holling's time budget arguments. Our BRC model overcomes several model construction problems in Courchamp et al. (1999), and admits richer, reasonable and realistic dynamics. We explore the possible control strategies to save or restore the bird by controlling or eliminating the rat or the cat when the bird is endangered. We establish the existence of two types of mesopredator release phenomena: severe mesopredator release, where once superpredators are suppressed, a burst of mesopredators follows which leads their shared prey to extinction; and mild mesopredator release, where the mesopredator release could assert more negative impact on the endemic prey but does not lead the endemic prey to extinction. A sharp sufficient criterion is established for the occurrence of severe mesopredator release. We also show that, in a prey-mesopredator-superpredator trophic food web, eradication of introduced superpredators such as feral domestic cats in the BRC model, is not always the best solution to protect endemic insular prey. The presence of a superpredator may have a beneficial effect in such systems. (C) 2005 Society for Mathematical Biology. Published by Elsevier Ltd. All rights reserved.
\end{abstract}

\footnotetext{
* Corresponding author. Tel.: +1 602965 6915; fax: +1 6029658119 .

E-mail addresses: mfan@nenu.edu.cn (M. Fan), kuang@asu.edu (Y. Kuang), zfeng@math.purdue.edu (Z. Feng).
} 


\section{Introduction}

Recent history indicates that the oceanic insular ecosystems are extremely sensitive to anthropogenic perturbation. Among those, successful introduction of alien species is a major cause of ecosystem perturbation (Williamson, 1996) on oceanic islands. Introduced predators frequently have a dramatic effect on native prey (Diamond, 1989), frequently the cause of native species extinction (Atkinson, 1989; Moors and Atkinson, 1984; Williamson, 1996). Many introduced species are highly adaptable, often find exceptionally good conditions in these islands (Apps, 1986), and encounter few pathogens and parasites (Dobson, 1988). Such introduced alien predation is the major cause of $42 \%$ of island bird extinctions in the past and a major factor endangering $40 \%$ of currently threatened island bird species (King, 1985). Among the most notorious and harmful introduced predators are feral cats Felis catus and mongooses Hagolestes auropunctatus. Cat and mongoose have often been introduced in attempts to control rats, which generally reach ashore from either hitching a ride on sealing or whaling boats or from shipwrecks (Moors and Atkinson, 1984), had a devastating effect on ground nesting birds and tree nesting birds, and prey on eggs, chicks and sometimes the adults (Stapp, 2002). However, these introduced predators often attack native prey (Diamond, 1989; Moors and Atkinson, 1984), which have no anti-predation mechanisms (Moors and Atkinson, 1984) and have not co-evolved with the introduced predator (this is particularly true for seabirds, which must return to land to raise their young, after nesting on islands). For example, feral cats are known to have been introduced into at least 65 islands group and to be a major threat to many island birds species (Atkinson, 1989), and are responsible for the loss of numerous land and seabird colonies, populations or even species (Atkinson, 1989; Rodriguez-Estrella et al., 1991). Cats also constitute a major threat to many endemic reptile species or subspecies (Arnaud et al., 1993; Case and Bolger, 1991; Iverson, 1978) and mammals (Mellink, 1992; Spencer, 1991).

The feral domestic cat is a opportunistic predator, eating what is most easily available, switching prey according to their relative spatial and temporal availability (Fitzgerald, 1988). When rats are more abundant than birds, reptiles or other mammals, the feral domestic cats are known to prey largely upon them, whereas rats can constitute a smaller part of the cat diet when birds, reptiles or other mammals are relatively more abundant (Bloomer and Bester, 1990; Nogales and Medina, 1996; Nogales et al., 1992).

Invasive alien species interact with other elements of global change to cause considerable damage to managed and natural systems and to incur huge costs in society (Mooney and Hobbs, 2000). Invasive species are a major cause of extinctions, second only to habitat destruction, and they exact a cost of approximately $\$ 120$ billion per year in the United States alone (Russo, 2004). In response, several measures have been developed and deployed to control, contain or eradicate a wide range of invasive species in affected areas (Zavaleta et al., 2001). Invasive species eradication is an increasingly important component of the conservation and management of natural ecosystems (Zavaleta et al., 2001). Where it is possible, eradication is the favored approach (Zavaleta et al., 2001).

The harm caused by the introduced predators on oceanic islands is widely known, and control programs are largely recognized as the best way to restore ecosystems (Atkinson, 1988). Control of alien species has been recommended by both theoretical and field 
conservationists. In many cases, introduced predators and preys both occur together. For example at least 80 islands have both alien cats and rats. Priority is then generally given to the control of the predators, since they have the most direct and obvious short term effects. Introduced feral cats have been the subject of many control attempts. Eradication of those alien cat populations is required in many cases, has often been tried and several cat eradication programmes are currently underway (e.g., Courchamp et al. (1999) and references cited therein). Successful cat eradication has been reported for several islands (Domm and Messersmith, 1990; Rauzon, 1985). However, the ecological context of eradication is very complex and there is also evidence that, without sufficient planning, successful eradications can have many undesired and unexpected impacts on native species and ecosystems. For example, 'Paradoxically, in some particular situations, the presence of a controlled population of cats might be, at least temporarily, more beneficial to their endemic prey than its eradication. Such is the case on many islands where rodents have also been introduced' (Courchamp et al., 1999). Recently, Zavaleta et al. (2001) deliberately discussed the possible secondary effects that result from the successful removal of invasive species.

One typical impact among those resulting from the successful removal of invasive species is the concept known to ecologists as mesopredator release (Soule et al., 1988) following superpredator (top predator) control or removal (an example of a top-down trophic cascade), which has been suggested as the cause of decline and extinction of some endemic prey species. For example, it has been shown that the rapid eradication of cats could trigger an explosive increase in the rat population after the removal, and then could lead to a more severe negative impact on the endemic species. Such a expansion of rat population would be more detrimental for endemic small vertebrates (Courchamp et al., 1999). Attempted reduction of the cat population of Amsterdam Island is alleged to have caused a compensating rise in the number of rats and mice, and so has been abandoned (Courchamp et al., 1999). In short, the threat of mesopredator release following superpredator eradication is very real and has been extensively reported (Courchamp et al., 1999; Crooks and Soule, 1999; Rogers and Caro, 1998; Rogers and Heard, 2000; Soule et al., 1988).

On the other hand, in some situations, the eradication of rodents first is necessary, which, historically thought to be impossible, has been proven feasible and is now being achieved regularly, even on relatively large islands (Taylor and Thomas, 1993; Towns, 1996). However, the eradication of rodents such as rats and rabbits can cause problems by forcing the superpredator (for example, cats) to switch prey, resulting in a brutal increase in predation pressure on the endemic prey species, as experienced by native stoats and rats in New Zealand (Murphy and Bradfield, 1992).

It is reported (Rogers and Heard, 2000) that the mesopredator release is most likely to occur in food webs characterized by (1) efficient predation with prey held well below resource limitation, (2) lack of extensive omnivory, and (3) either low diversity of top predators, or all top predators removed together. These conditions are generally met by the landbird-mesopredator-superpredator system, in which a top predator feeds on two species, one of which is also a predator on the third (Rogers and Heard, 2000). Although many field conservationists are now aware of the existence of such complex relationships, theoretical demonstration and characterization is still lacking. In 1999, Courchamp et al. (1999) constructed a mathematical model of the interactions among a prey species, 
a mesopredator species and a superpredator species, which are referred to below as bird, rat and cat, respectively. The model is governed by the following system of ordinary differential equations:

$$
\begin{aligned}
\frac{\mathrm{d} B}{\mathrm{~d} t} & =r_{b} B\left(1-\frac{B}{K_{b}}\right)-\frac{B}{S+B} \eta_{b} R-\frac{B}{B+R} \mu_{b} C, \\
\frac{\mathrm{d} R}{\mathrm{~d} t} & =r_{r} R\left(1-\frac{\eta_{b} \eta_{s} R}{\eta_{b} S+\eta_{s} B}\right)-\frac{R}{B+R} \mu_{r} C, \\
\frac{\mathrm{d} C}{\mathrm{~d} t} & =r_{c} C\left(1-\frac{\mu_{b} \mu_{r} C}{\mu_{r} B+\mu_{b} R}\right),
\end{aligned}
$$

where it is assumed that the superpredator preys upon both the prey and the mesopredator, and

- $B(t), R(t)$ and $C(t)$ denote the number of individuals at time $t$ in the prey, mesopredator and superpredator populations, respectively;

- $S$ represents the quantity of non-avian food for mesopredators;

- $r_{b}, r_{r}$ and $r_{c}$ are the intrinsic growth rate of the prey, mesopredator, and superpredator, respectively;

- $\eta_{b}$ and $\eta_{s}$ are the predation rate of the mesopredator on the prey and on other food items (denoted by $S$ ) such as seeds, leaves, invertebrates;

- $K_{b}$ is the carrying capacity of the environment for the prey population;

- $\mu_{b}$ and $\mu_{r}$ are the predation rate of the superpredator on the prey and the mesopredator.

This theoretical work presents several interesting features of direct concern to conservation biology:

Conclusion 1. The presence of one predator only is sufficient to induce the extinction of the endemic prey.

Conclusion 2. When both the mesopredator and the superpredator are present, several situations may arise, among which is the case where the three species are present with stable dynamics. Another interesting case is that both predator species can coexist indefinitely, even after the eradication of the prey species.

Conclusion 3. The superpredator causes the extinction of the mesopredator, but not of the prey. The presence of the superpredator may preclude the elimination of the prey by the mesopredator. In addition, the authors of Courchamp et al. (1999) proposed that the superpredator eradication practice should be carefully studied before its implementation to prevent the mesopredator release effect.

Although the conclusions are plausible, the model formulation of (1.1) is problematic. For example, if the prey species is absent in the three-species system, then (1.1) reduces to

$$
\begin{aligned}
& \frac{\mathrm{d} R}{\mathrm{~d} t}=r_{r} R\left(1-\frac{\eta_{s} R}{S}\right)-\mu_{r} C, \\
& \frac{\mathrm{d} C}{\mathrm{~d} t}=r_{c} C\left(1-\frac{\mu_{r} C}{R}\right),
\end{aligned}
$$

where the predation of the superpredator on the mesopredator depends only on the size of the superpredator which is not natural or realistic. This situation also occurs in the case when the mesopredator is absent in (1.1). 
Another serious drawback of (1.1) is that it fails to model the interference between predator individuals, which has been suggested by large numbers of field and laboratory experiments and observations (e.g. Dolman (1995)). One significant component of the predator-prey interaction is the predator's rate of feeding upon prey, namely the so-called predator's functional responses. The consumption process is potentially influenced by many factors; it is unlikely that one mathematical expression can describe the function response in all systems. There are about 30 proposed equations for the functional response (collections in Jost (1998)). In (1.1), the predator's functional responses are just prey-dependent, i.e., $B /(S+B)$ (predation rate by rat) and $B /(B+R)$ (predation rate by cat) in the prey equation, and $R /(B+R)$ (predation by cat) in the mesopredator population, which fails to model the interference between the predators.

In order to model the predators' interference, the functional response must be predator dependent. Many empirical and statistical studies (Ariditi et al., 1991; Dolman, 1995; Skalski and Gilliam, 2001) show that predator dependence in the functional response is very frequent in laboratory and natural systems. Jost and Ellner (2000) carried out systematically statistical studies and obtained significant evidence of predator dependence in the functional response. Theoretical studies have shown that the dynamics of models with predator-dependent functional responses can differ considerably from those with preydependent functional responses (Fan and Kuang, 2004).

Oscillatory population dynamics is often observed in ecosystems that are subjected to human disturbance (Hwang and Kuang, 2003). From this perspective, another possible limitation of (1.1) is the lack of oscillatory dynamics.

Although the model (1.1) is problematic, the authors of Courchamp et al. (1999) noted the possible occurrence of the mesopredator release in its dynamics and gave the sufficient criterion for that to happen in term of the control efforts. The authors of Courchamp et al. (1999) also touched on the discussion of the control strategy to protect the birds. However, the following important questions are yet to be answered:

Question A: For a prey-mesopredator-superpredator system (without control), when can the mesopredator release occur?

Question B: When it does occur, can it cause the extinction of the endemic prey?

In this paper, we will (1): develop a more realistic model of the prey-mesopredatorsuperpredator interactions; (2): obtain explicit conditions for the occurrence of the mesopredator release phenomenon; (3): design control strategies to effectively restore endangered endemic preys.

The rest of this paper is organized as follows. In Section 2, we derive the rat's and the cat's functional responses based on the classical Holling's time budget argument and formulate a prey-mesopredator-superpredator model. Section 3 focuses on the equilibria dynamics of this model. In Section 4, we systematically investigate several different control strategies to protect or to restore the endangered native bird species. In particular, we establish the existence of two types of mesopredator release phenomena in our model: severe mesopredator release, where once superpredators are suppressed, a burst of mesopredators follows which leads their shared prey to extinction; and moderate mesopredator release, where the mesopredator release could assert more negative impact on the endemic prey but does not lead the endemic prey to extinction. A sharp sufficient 
criterion is established for the occurrence of severe mesopredator release. We also show that, in a prey-mesopredator-superpredator trophic food web, eradication of introduced superpredators such as feral domestic cats in the BRC model, is not always the best solution to protect endemic insular prey. In the discussion section, we examine additional biological implications of our mathematical findings and state some biologically motivated mathematical questions for future study.

Throughout this paper, extensive computational results are presented to illustrate or complement our mathematical observations and findings.

\section{Derivation of the model}

For convenience, as in Courchamp et al. (1999), we will frequently refer to the indigenous prey population as the bird population, refer to the mesopredator population as the rat population, and refer to the superpredator population as the cat population. For simplicity, we will often refer to them as bird, rat and cat, instead of prey, mesopredator and superpredator, respectively.

First, we derive suitable candidates for the rat's and the cat's functional responses. We will follow a classical Holling time budget argument (Beddington, 1975; Holling, 1965).

\subsection{Cat's functional response}

We assume that the total time spent by a cat for gathering food from both bird and rat is $T$, which can be divided into four parts:

- $T_{c s}$ : the time spent by cat for searching bird and rat.

- $T_{c h b}$ : the time spent by cat for handling caught bird.

- $T_{c h r}$ : the time spent by cat for handling caught rat.

- $T_{c w}$ : the time wasted by cat for interfering with other cat.

The number of birds (rats) caught per cat is then proportional to the bird (rat) density $B(R)$ and the search time. Then

- the total number of birds caught per cat $\left(N_{c b}\right)=\alpha_{c b} B T_{c s}$,

- the total number of rats caught per cat $\left(N_{c r}\right)=\alpha_{c r} R T_{c s}$,

where

- $\alpha_{c b}$ is the searching efficiency of cat for bird,

- $\alpha_{c r}$ is the searching efficiency of cat for rat.

The cat has a series of choices when it captures prey: it may kill and consume the prey immediately; or it may 'play' with the prey before killing or eating it; or it might also catch prey to eat later (Fitzgerald and Turner, 2000). For simplicity, on average, we assume that the total time wasted for handling caught birds (rats) is equal to the product of the total number of caught birds (rats) and the expected handling time $t_{c h b}\left(t_{c h r}\right)$ per unit bird (rat), i.e.,

$$
T_{c h b}=\alpha_{c b} t_{c h b} B T_{c s}, \quad T_{c h r}=\alpha_{c r} t_{c h r} R T_{c s} .
$$


The wasted time $T_{c w}$ can be partitioned further so that it is given by the product of the number of encounters between cats $\left(N_{c e}\right)$ and the time wasted per encounter $t_{c w}$. Generally, cats living on dispersed natural prey do not form groups and typically live alone (Liberg et al., 2000). If the cats move randomly, $N_{c e}$ can be expressed as

$$
N_{c e}=\alpha_{c c} T_{c s}(C-1),
$$

where $\alpha_{c c}$ is the rate of encounter between cats, related to both their speed of movement and the range at which they sense each other. The total time wasted due to encounters between cats is then given by

$$
T_{c w}=\alpha_{c c} T_{c s}(C-1) t_{c w} .
$$

Hence,

$$
T=T_{c s}+T_{c h b}+T_{c h r}+T_{c w}=\left[1+\alpha_{c b} t_{c h b} B+\alpha_{c r} t_{c h r} R+\alpha_{c c} t_{c w}(C-1)\right] T_{c s} .
$$

Consequently, the cat's functional responses are

$$
\begin{aligned}
\frac{N_{c b}}{T} & =\frac{\alpha_{c b} B}{1+\alpha_{c b} t_{c h b} B+\alpha_{c r} t_{c h r} R+\alpha_{c c} t_{c w}(C-1)}, \\
\frac{N_{c r}}{T} & =\frac{\alpha_{c r} R}{1+\alpha_{c b} t_{c h b} B+\alpha_{c r} t_{c h r} R+\alpha_{c c} t_{c w}(C-1)} .
\end{aligned}
$$

\subsection{Rat's functional response}

When there is no bird, rat can live on other food items such as seeds, leaves, invertebrates and so on. In the following, we assume that the quantity of such non-avian food is a constant $S$. The derivation of the rat's functional response is similar to that of the cat's. The parameters used to derive the rat's functional response and their biological meanings are listed in the following table.

\begin{tabular}{ll}
\hline Parameters & Biological definition \\
\hline$T$ & the rat's total time spent acquiring bird and non-avian food \\
$T_{r s}$ & the time spent by rat for searching bird and non-avian food \\
$T_{r h b}$ & the time spent by rat for handling caught bird \\
$T_{r h f}$ & the time spent by rat for handling non-avian food \\
$T_{r w}$ & the time wasted for interfering with other rats \\
$\alpha_{r b}$ & the searching efficiency rate of rat for bird \\
$\alpha_{r f}$ & the searching efficiency rate of rat for non-avian food \\
$\alpha_{r r}$ & the rate of encounter between rats \\
$N_{r b}$ & the total number of bird caught per rat \\
$N_{r f}$ & the total amount of non-avian food caught per rat \\
$N_{r e}$ & the number of encounters between rats \\
$t_{r w}$ & the time wasted per encounter of rat \\
$t_{r h b}$ & the expected handling time per unit bird \\
$t_{r h f}$ & the expected handling time per unit non-avian food \\
\hline
\end{tabular}

It is easy to observe the following.

$$
N_{r b}=\alpha_{r b} B T_{r s}, \quad N_{r f}=\alpha_{r f} S T_{r s},
$$




$$
\begin{aligned}
& T_{r h b}=\alpha_{r b} t_{r h b} B T_{r s}, \quad T_{r h f}=\alpha_{r f} t_{r h f} S T_{r s}, \\
& T_{r w}=N_{r e} t_{c w}=\alpha_{r r} T_{r s}(R-1) t_{c w} .
\end{aligned}
$$

The total time of a rat spent preying bird and non-avian food is

$$
T=T_{r s}+T_{r h b}+T_{r h f}+T_{r w}=\left(1+\alpha_{r b} t_{r h b} B+\alpha_{r f} t_{r h f} S+\alpha_{r r} t_{c w}(R-1)\right) T_{r s} .
$$

By carrying out similar arguments as in the previous subsection, one can obtain the following rat's functional responses:

$$
\begin{aligned}
\frac{N_{r b}}{T} & =\frac{\alpha_{r b} B}{1+\alpha_{r b} t_{r h b} B+\alpha_{r f} t_{r h f} S+\alpha_{r r} t_{c w}(R-1)}, \\
\frac{N_{r f}}{T} & =\frac{\alpha_{r f} S}{1+\alpha_{r b} t_{r h b} B+\alpha_{r f} t_{r h f} S+\alpha_{r r} t_{c w}(R-1)} .
\end{aligned}
$$

\subsection{The model}

Let $B(t), R(t), C(t)$ be the densities of the birds, the rats and the cats, respectively. In the absence of rats and cats, we assume that the birds' growth obeys the simple logistic growth law,

$$
\frac{\mathrm{d} B}{\mathrm{~d} t}=r B\left(1-\frac{B}{K}\right),
$$

where $r$ and $K$ stand for the intrinsic growth rate and the environmental carrying capacity of the birds, respectively. In the absence of any preys, the rats and the cats grow (decline) exponentially:

$$
\frac{\mathrm{d} R}{\mathrm{~d} t}=-d_{r} R, \quad \frac{\mathrm{d} C}{\mathrm{~d} t}=-d_{c} C,
$$

where $d_{r}$ and $d_{c}$ are the death rates of the rats and the birds respectively. In the following, we assume that rats and cats consume the birds, the rats consume the non-avian food, and the cats consume the rats according to the functional responses derived in the previous subsections. Let $\eta_{r b}, \eta_{r f}, \eta_{c b}$ and $\eta_{c r}$ be the conversion rates of prey biomass into that of the corresponding predators. We obtain the following BRC model:

$$
\begin{aligned}
\frac{\mathrm{d} B}{\mathrm{~d} t} & =r B\left(1-\frac{B}{K}\right)-\frac{\alpha_{r b} B R}{F(B, S, R)}-\frac{\alpha_{c b} B C}{G(B, R, C)}, \\
\frac{\mathrm{d} R}{\mathrm{~d} t} & =\frac{\eta_{r b} \alpha_{r b} B R+\eta_{r f} \alpha_{r f} S R}{F(B, S, R)}-d_{r} R-\frac{\alpha_{c r} R C}{G(B, R, C)}, \\
\frac{\mathrm{d} C}{\mathrm{~d} t} & =-d_{c} C+\frac{\eta_{c b} \alpha_{c b} B C+\eta_{c r} \alpha_{c r} R C}{G(B, R, C)},
\end{aligned}
$$

where

$$
\begin{aligned}
& F(B, S, R)=1+\alpha_{r b} t_{r h b} B+\alpha_{r f} t_{r h f} S+\alpha_{r r} t_{c w}(R-1), \\
& G(B, R, C)=1+\alpha_{c b} t_{c h b} B+\alpha_{c r} t_{c h r} R+\alpha_{c c} t_{c w}(C-1) .
\end{aligned}
$$


For simplicity, we rescale the parameters in (2.1) by the following substitutions:

$$
\begin{aligned}
& a_{1}=\frac{\alpha_{r b}}{1+\alpha_{r f} t_{r h f} S-\alpha_{r r} t_{c w}}, \quad \delta=\frac{\alpha_{r b} t_{r h b}}{1+\alpha_{r f} t_{r h f} S-\alpha_{r r} t_{c w}}, \\
& \sigma=\frac{\alpha_{r r} t_{c w}}{1+\alpha_{r f} t_{r h f} S-\alpha_{r r} t_{c w}} \\
& a_{2}=\frac{\alpha_{c b}}{1-\alpha_{c c} t_{c w}}, \quad c_{1}=\frac{\eta_{c b} \alpha_{c b}}{1-\alpha_{c c} t_{c w}}, \quad c_{2}=\frac{\eta_{c r} \alpha_{c r}}{1-\alpha_{c c} t_{c w}}, \\
& b_{1}=\frac{\eta_{r b} \alpha_{r b}}{1+\alpha_{r f} t_{r h f} S-\alpha_{r r} t_{c w}}, \quad b_{2}=\frac{\eta_{r f} S}{1+\alpha_{r f} t_{r h f} S-\alpha_{r r} t_{c w}}, \\
& b_{3}=\frac{\alpha_{c r}}{1-\alpha_{c c} t_{c w}} \\
& \alpha=\frac{\alpha_{c b} t_{c h b}}{1-\alpha_{c c} t_{c w}}, \quad \beta=\frac{\alpha_{c r} t_{c h r}}{1-\alpha_{c c} t_{c w}}, \quad \gamma=\frac{\alpha_{c c} t_{c w}}{1-\alpha_{c c} t_{c w}} .
\end{aligned}
$$

Then, the system takes the form

$$
\begin{aligned}
\frac{\mathrm{d} B}{\mathrm{~d} t} & =r B\left(1-\frac{B}{K}\right)-\frac{a_{1} B R}{1+\delta B+\sigma R}-\frac{a_{2} B C}{1+\alpha B+\beta R+\gamma C} \\
\frac{\mathrm{d} R}{\mathrm{~d} t} & =\frac{b_{1} B R+b_{2} R}{1+\delta B+\sigma R}-d_{r} R-\frac{b_{3} R C}{1+\alpha B+\beta R+\gamma C} \\
\frac{\mathrm{d} C}{\mathrm{~d} t} & =-d_{c} C+\frac{c_{1} B C+c_{2} R C}{1+\alpha B+\beta R+\gamma C} .
\end{aligned}
$$

If the bird and cat species are absent in (2.2), the rat equation reduces to

$$
\frac{\mathrm{d} R}{\mathrm{~d} t}=\frac{b_{2} R}{1+\sigma R}-d_{r} R
$$

which is, mathematically, the same as the Smith single species growth model (Smith, 1963). If the rat or the cat is absent in (2.2), then (2.2) reduces to the so-called predator-prey system with Beddington-DeAngelis functional response, which has been studied extensively in the literature (Fan and Kuang, 2004; Hwang, 2003, 2004).

To ensure biological relevance, we will assume that $B(0)>0, R(0)>0, C(0)>0$ and all the parameters in (2.2) are positive.

\section{Equilibria and their local stability}

Before our systematic analysis of (2.2), we present below some preliminary results such as positivity, existence, and uniqueness without proof. They follow from some careful but standard mathematical arguments.

Lemma 3.1. For any $B(0)>0, R(0)>0, C(0)>0,(2.2)$ has a unique positive solution defined for all $t \geq 0$.

We now examine the condition for the existence and local stability of boundary and positive equilibria. The possible equilibria or steady states of (2.2) are listed below:

$$
E_{0}:(0,0,0), E_{b}:(K, 0,0), E_{r}:\left(0, \frac{b_{2}-d_{r}}{\sigma d_{r}}, 0\right),
$$


$E_{r c}:\left(0, R_{3}^{*}, C_{3}^{*}\right)$, where $R_{3}^{*}$ and $C_{3}^{*}$ solve

$$
\frac{b_{2}}{1+\sigma R}-d_{r}-\frac{b_{3} C}{1+\beta R+\gamma C}=0 \quad \frac{c_{2} R}{1+\beta R+\gamma C}-d_{c}=0 ;
$$

$E_{b c}:\left(B_{4}^{*}, 0, C_{4}^{*}\right)$, where $B_{4}^{*}$ and $C_{4}^{*}$ solve

$$
r(1-B / K)-\frac{a_{2} C}{1+\alpha B+\gamma C}=0, \quad \frac{c_{1} B}{1+\alpha B+\gamma C}-d_{c}=0 ;
$$

$E_{b r}:\left(B_{5}^{*}, R_{5}^{*}, 0\right)$, where $B_{5}^{*}$ and $R_{5}^{*}$ solve

$$
r(1-B / K)-\frac{a_{1} R}{1+\delta B+\sigma R}=0, \quad \frac{b_{1} B+b_{2}}{1+\delta B+\sigma R}-d_{r}=0 ;
$$

$E_{b r c}:\left(B_{6}^{*}, R_{6}^{*}, C_{6}^{*}\right)$, where $B_{6}^{*}, R_{6}^{*}$ and $C_{6}^{*}$ solve

$$
\begin{aligned}
& r(1-B / K)-\frac{a_{1} R}{1+\delta B+\sigma R}-\frac{a_{2} C}{1+\alpha B+\beta R+\gamma C}=0 \\
& \frac{b_{1} B+b_{2}}{1+\delta B+\sigma R}-d_{r}-\frac{b_{3} C}{1+\alpha B+\beta R+\gamma C}=0 \\
& \frac{c_{1} B+c_{2} R}{1+\alpha B+\beta R+\gamma C}-d_{c}=0 .
\end{aligned}
$$

In order to determine the local stability of these equilibria, we calculate the variational matrix of (2.2). After some straightforward algebraic calculations, we obtain

$$
J(B, R, C)=\left(a_{i j}\right)_{3 \times 3},
$$

where

$$
\begin{aligned}
& a_{11}=r\left(1-\frac{2 B}{K}\right)-\frac{a_{1} R(1+\sigma R)}{(1+\delta B+\sigma R)^{2}}-\frac{a_{2} C(1+\beta R+\gamma C)}{(1+\alpha B+\beta R+\gamma C)^{2}}, \\
& a_{12}=-\frac{a_{1} B(1+\delta B)}{(1+\delta B+\sigma R)^{2}}+\frac{a_{2} \beta B C}{(1+\alpha B+\beta R+\gamma C)^{2}}, \\
& a_{13}=-\frac{a_{2} B(1+\alpha B+\beta R)}{(1+\alpha B+\beta R+\gamma C)^{2}}, \\
& a_{21}=\frac{b_{1} R(1+\sigma R)-\delta b_{2} R}{(1+\delta B+\sigma R)^{2}}+\frac{\alpha b_{3} R C}{(1+\alpha B+\beta R+\gamma C)^{2}}, \\
& a_{22}=\frac{\left(b_{1} B+b_{2}\right)(1+\delta B)}{(1+\delta B+\sigma R)^{2}}-d_{r}-\frac{b_{3} C(1+\alpha B+\gamma C)}{(1+\alpha B+\beta R+\gamma C)^{2}}, \\
& a_{23}=-\frac{b_{3} R(1+\alpha B+\beta R)}{(1+\alpha B+\beta R+\gamma C)^{2}}, \\
& a_{32}=\frac{c_{2} C(1+\alpha B+\gamma C)-\beta c_{1} B C}{(1+\alpha B+\beta R+\gamma C)^{2}}, \\
& a_{33}=-d_{c}+\frac{\left(c_{1} B+c_{2} R\right)(1+\alpha B+\beta R)}{(1+\alpha B+\beta R+\gamma C)^{2}} .
\end{aligned}
$$


The stability of equilibria $\left(B^{*}, R^{*}, C^{*}\right)$ is determined by the eigenvalues of the matrix $J\left(B^{*}, R^{*}, C^{*}\right)$. For $E_{0}$, we have

$$
J\left(E_{0}\right)=J(0,0,0)=\left(\begin{array}{ccc}
r & 0 & 0 \\
0 & -d_{r} & 0 \\
0 & 0 & -d_{c}
\end{array}\right) .
$$

This yields the following theorem.

Theorem 3.1. $\left(E_{0}\right)$ is always a saddle node and there cannot be total extinction of the system (2.2) for positive initial conditions.

Theorem 3.2. $E_{b}=(K, 0,0)$ always exists and is stable if

$$
\begin{aligned}
& d_{r}>\frac{b_{1} K+b_{2}}{1+\delta K}, \quad d_{c}>\frac{c_{1} K}{1+\alpha K} . \\
E_{r}= & \left(0,\left(b_{2}-d_{r}\right) /\left(\sigma d_{r}\right), 0\right) \text { exists if } b_{2}>d_{r} \text { and it is stable if } \\
& 0<d_{r}<b_{2}\left(1-\frac{r \sigma}{a_{1}}\right), \quad d_{c}>\frac{c_{2}\left(b_{2}-d_{r}\right)}{\sigma d_{r}+\beta\left(b_{2}-d_{r}\right)} .
\end{aligned}
$$

Proof. The conclusion directly follows from the fact that

$$
J\left(E_{b}\right)=\left(\begin{array}{ccc}
-\frac{r}{K} & -\frac{a_{1} K}{1+\delta K} & -\frac{a_{2} K}{1+\alpha K} \\
0 & \frac{b_{1} K+b_{2}}{1+\delta K}-d_{r} & 0 \\
0 & 0 & -d_{c}+\frac{c_{1} K}{1+\alpha K}
\end{array}\right)
$$

and

$$
J\left(E_{r}\right)=\left(\begin{array}{ccc}
r-\frac{a_{1}\left(b_{2}-d_{r}\right)}{\sigma b_{2}} & 0 & 0 \\
\frac{\left(b_{1}-\delta b_{r}\right)\left(b_{2}-d_{r}\right)}{\sigma b_{2}} & \frac{d_{r}\left(d_{r}-b_{2}\right)}{b_{2}} & -\frac{b_{3}\left(b_{2}-d_{r}\right)}{\sigma d_{r}+\beta\left(b_{2}-d_{r}\right)} \\
0 & 0 & -d_{c}+\frac{c_{2}\left(b_{2}-d_{r}\right)}{\sigma d_{r}+\beta\left(b_{2}-d_{r}\right)}
\end{array}\right) .
$$

Theorem 3.3. Assume that

$$
d_{r}<b_{2}, \quad 0<d_{c}<\frac{c_{2}\left(b_{2}-d_{r}\right)}{\sigma d_{r}+\beta\left(b_{2}-d_{r}\right)},
$$

then $E_{r c}$ exists, where

$$
\frac{d_{c}}{c_{2}-\beta d_{c}}<R_{3}^{*}<\frac{b_{2}-d_{r}}{\sigma d_{r}} .
$$

Moreover, if

$$
c_{2} \gamma \geq b_{3} \beta, \quad 0<d_{r}<\min \left\{b_{2}, \frac{a_{1} b_{3}}{\sigma a_{2}}\right\}-\frac{r b_{3}}{a_{2}},
$$

then $E_{r c}$ is locally asymptotically stable. 
Proof. Note that $E_{r c}$ solves (3.1). Under the above assumption, one can show that the following curves

$$
C=\frac{c_{2}\left(b_{2}-d_{r}\right) R-\sigma d_{4} c_{2} R^{2}}{b_{3} d_{c}(1+\sigma R)}, \quad C=\frac{\left(c_{2}-\beta d_{c}\right) R}{\gamma d_{c}}-\frac{1}{\gamma}
$$

intersect each other in the first quadrant of the $R-C$ coordinate system and the coordinates of the unique intersection are the last two components of $E_{r c}$.

With the help of (3.1), (3.5) and (3.6), after doing some simple algebraic calculation, we obtain

$$
J\left(E_{r c}\right)=\left(\begin{array}{ccc}
a_{11} & 0 & 0 \\
a_{21} & a_{22} & a_{23} \\
a_{31} & a_{32} & a_{33}
\end{array}\right)
$$

where

$$
\begin{aligned}
& a_{11}=r-\frac{a_{1} R_{3}^{*}}{1+\sigma R_{3}^{*}}-\frac{a_{2} C_{3}^{*}}{1+\beta R_{3}^{*}+\gamma C_{3}^{*}}, \\
& a_{21}=\frac{b_{1} R_{3}^{*}\left(1+\sigma R_{3}^{*}\right)-\delta b_{2} R_{3}^{*}}{\left(1+\sigma R_{3}^{*}\right)^{2}}+\frac{\alpha b_{3} R_{3}^{*} C_{3}^{*}}{\left(1+\beta R_{3}^{*}+\gamma C_{3}^{*}\right)^{2}}, \\
& a_{22}=-\frac{b_{2} \sigma R_{3}^{*}}{\left(1+\sigma R_{3}^{*}\right)^{2}}+\frac{b_{3} \beta R_{3}^{*} C_{3}^{*}}{\left(1+\beta R_{3}^{*}+\gamma C_{3}^{*}\right)^{2}}, \quad a_{23}=-\frac{b_{3} R_{3}^{*}\left(1+\beta R_{3}^{*}\right)}{\left(1+\beta R_{3}^{*}+\gamma C_{3}^{*}\right)^{2}}, \\
& a_{31}=\frac{c_{1} C_{3}^{*}\left(1+\beta R_{3}^{*}+\gamma C_{3}^{*}\right)-\alpha c_{2} R_{3}^{*} C_{3}^{*}}{\left(1+\beta R_{3}^{*}+\gamma C_{3}^{*}\right)^{2}}, \quad a_{32}=\frac{c_{2} C_{3}^{*}\left(1+\gamma C_{3}^{*}\right)}{\left(1+\beta R_{3}^{*}+\gamma C_{3}^{*}\right)^{2}}, \\
& a_{33}=-\frac{c_{2} \gamma R_{3}^{*} C_{3}^{*}}{\left(1+\beta R_{3}^{*}+\gamma C_{3}^{*}\right)^{2}} .
\end{aligned}
$$

We have

$$
\begin{aligned}
& a_{11}=\frac{\left(r b_{3}-a_{2} b_{2}+a_{2} d_{r}\right)+\left(r b_{3} \sigma-a_{1} b_{3}+a_{2} d_{r} \sigma\right) R_{3}^{*}}{b_{3}\left(1+\sigma R_{3}^{*}\right)}<0, \\
& a_{22}+a_{33}=-\frac{b_{2} \sigma R_{3}^{*}}{\left(1+\sigma R_{3}^{*}\right)^{2}}+\frac{\left(b_{3} \beta-c_{2} \gamma\right) R_{3}^{*} C_{3}^{*}}{\left(1+\beta R_{3}^{*}+\gamma C_{3}^{*}\right)^{2}}<0, \\
& a_{22} a_{33}-a_{32} a_{23}=\frac{b_{2} c_{2} \sigma \gamma R_{3}^{* 2} C_{3}^{*}}{\left(1+\sigma R_{3}^{*}\right)^{2}\left(1+\beta R_{3}^{*}+\gamma C_{3}^{*}\right)^{2}}+\frac{b_{3} c_{2} R_{3}^{*} C_{3}^{*}}{\left(1+\beta R_{3}^{*}+\gamma C_{3}^{*}\right)^{3}}>0,
\end{aligned}
$$

which implies that all the three roots of the characteristic equation of $J\left(E_{r c}\right)$ have negative real parts. Therefore, $E_{r c}$ is locally asymptotically stable.

By carrying out similar arguments as that for $E_{r c}$, we derive the following sufficient criteria for the existence and local asymptotical stability of $E_{b c}$ and $E_{b r}$.

Theorem 3.4. Assume that

$$
0<d_{c}<\frac{c_{1} K}{1+\alpha K}
$$


then $E_{b c}$ exists, where

$$
\frac{d_{c}}{c_{1}-\alpha d_{c}}<B_{4}^{*}<K
$$

Moreover, if

$$
c_{1} \gamma \geq \alpha a_{2}, \quad d_{r}>\max \left\{b_{2}-\frac{b_{3} r}{a_{2}}, \frac{K a_{2} b_{1}+a_{2} b_{2}-b_{3} r}{K a_{2} \delta+a_{2}}\right\},
$$

then $E_{b c}$ is locally asymptotically stable.

Theorem 3.5. Assume that

$$
b_{2}<d_{r}<\frac{b_{1} K+b_{2}}{1+\delta K} \quad \text { or } \quad 0<b_{2}\left(1-\frac{r \sigma}{a_{1}}\right)<d_{r}<\min \left\{b_{2}, \frac{b_{1}}{\delta}\right\}
$$

then $E_{b r}$ exists. Moreover, if

$$
a_{1} \delta \leq b_{1} \sigma, \quad b_{1}>\delta b_{2}, \quad d_{c} \geq \max \left\{\frac{c_{1}}{\alpha}, \frac{c_{2}}{\beta}\right\},
$$

then $E_{b r}$ exists and is locally asymptotically stable.

These theorems indicate that the simultaneous extinction of all the three populations will never occur since $E_{0}$ is unstable. The bird population tends to its carrying capacity in the absence of the alien populations $E_{b}$ while the rat tends to its carrying capacity $E_{r}$ when there are no birds and cats. The more interesting scenarios include the possible disappearance of the bird only $\left(E_{r c}\right)$, or the rat only $\left(E_{b c}\right)$, or the cat only $\left(E_{b r}\right)$. Numerical simulations show that the bird, rat and cat can coexist together at an equilibrium $E_{b r c}$ (see Fig. 3.1(b)); the explicit expressions of its components are too complex to be presented here (one can obtain them with the help of a Maple program). For the same reason, the stability criteria for $E_{b r c}$ are also too complex to be presented. However, the deterministic nature of the model allows us to carry out a systematic numerical study.

In addition to the above equilibrium dynamics, the system (2.2) can admit cyclic dynamics. In some cases, the attractor can be a boundary periodic solution $P_{b c}$ : $\left(B_{4}^{*}(t), 0, C_{4}^{*}(t)\right)$ (see Fig. 3.1(a)), while in some other cases, the attractor can be a positive periodic solution $P_{b r c}:\left(B_{6}^{*}(t), R_{6}^{*}(t), C_{6}^{*}(t)\right)$ (see Fig. 3.1(c)). The dynamics of (2.2) is summarized in Table 1.

Remark 3.1. In this section, we explored only the local stability of various equilibria. The global dynamics of model (2.2) is complex and very difficult to study analytically. As mentioned above, the subsystem Bird-Rat $C(t)=0$ and Bird-Cat, $R(t)=0$ are of Beddington-DeAngelis type. For these subsystems, the complete and elegant global results of Hwang $(2003,2004)$ apply.

Remark 3.2. Extensive numerical simulations suggest that both the boundary periodic solutions and the positive periodic solutions come from Hope bifurcations (see Fig. 3.3). However, this is not obvious mathematically. 
(a)

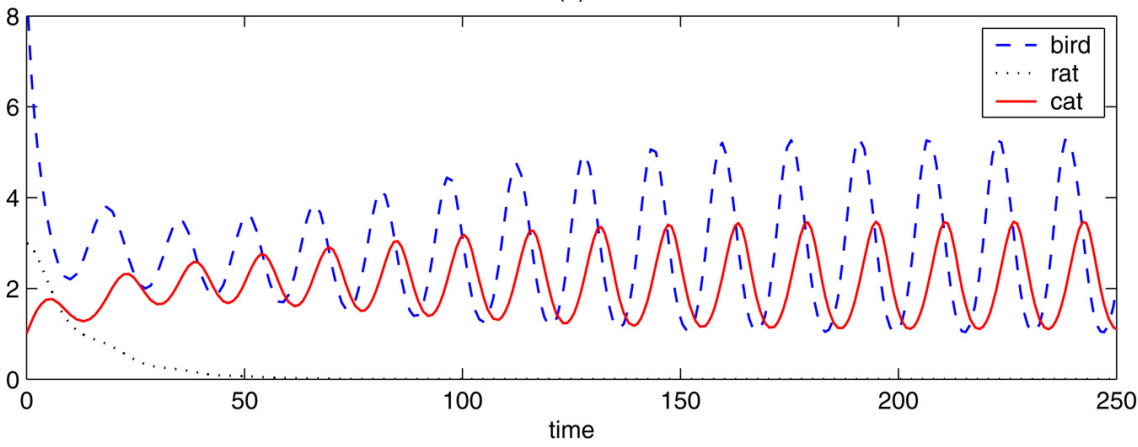

(b)

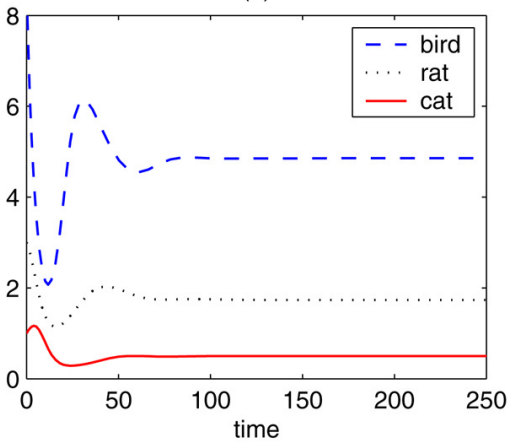

(c)

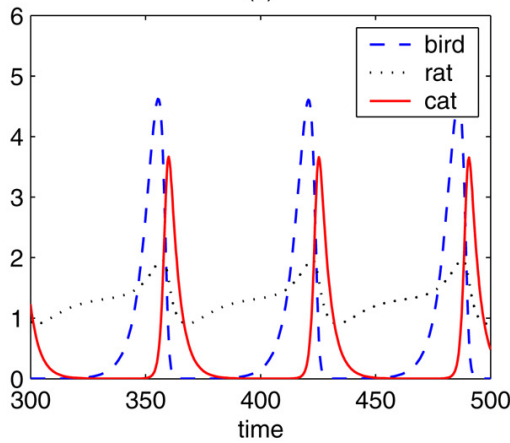

Fig. 3.1. Possible outcomes of (2.2): (a) $P_{b c}$ is an attractor, where the rat becomes extinct and the bird and the cat coexist cyclicly. $\left(r=1, K=10, a_{1}=0.7, a_{2}=1, \delta=0.5, \sigma=0.5, \alpha=0.6, \beta=0.4, \gamma=0.2, b 1=0.2\right.$, $\left.b_{2}=0.5, d_{r}=0.3, b_{3}=0.3, d_{c}=0.6, c_{1}=0.7, c_{2}=0.1\right)(\mathrm{b}) E_{b r c}$ is an attractor, where the bird, rat and cat coexist at equilibrium $\left(r=0.5, K=10, a_{1}=0.4, a_{2}=0.9, \delta=0.5, \sigma=0.5, \alpha=0.6, \beta=0.4, \gamma=0.2\right.$, $b_{1}=0.2, b_{2}=0.5, b_{3}=0.4, c_{1}=0.5, c_{2}=0.5, d_{r}=0.3, d_{c}=0.7$ ) (c) $P_{b r c}$ is an attractor, where the bird, rat and cat coexist cyclicly $\left(r=0.5, K=10, a_{1}=0.4, a_{2}=1, \delta=0.5, \sigma=0.5, \alpha=0.6, \beta=0.4, \gamma=0.2\right.$, $\left.b_{1}=0.2, b_{2}=0.5, d_{r}=0.3, b_{3}=0.1, d_{c}=0.3, c_{1}=1, c_{2}=0.1\right)$.

In the following, we depict the parameter ranges for the dynamic scenarios of (2.2) in the $d_{r}-d_{c}$ plane. Define

$$
\begin{aligned}
\Delta_{b} & =\left\{\left(d_{r}, d_{c}\right): d_{r}>\frac{b_{1} K+b_{2}}{1+\delta K}, \quad d_{c}>\frac{c_{1} K}{1+\alpha K}\right\}, \\
\Delta_{b c} & =\left\{\left(d_{r}, d_{c}\right): c_{1} \gamma \geq \alpha a_{2}, d_{r}>\max \left\{b_{2}-\frac{b_{3} r}{a_{2}}, \frac{K a_{2} b_{1}+a_{2} b_{2}-b_{3} r}{K a_{2} \delta+a_{2}}\right\},\right. \\
0 & \left.<d_{c}<\frac{c_{1} K}{1+\alpha K}\right\}, \\
\Delta_{b r} & =\left\{\left(d_{r}, d_{c}\right): a_{1} \delta \leq b_{1} \sigma, b_{1} \geq \delta b_{2}, b_{2}\left(1-\frac{r \sigma}{a_{1}}\right)<d_{r}<\frac{b_{1} K+b_{2}}{1+\delta K},\right. \\
d_{c} & \left.\geq \max \left\{\frac{c_{1}}{\alpha}, \frac{c_{2}}{\beta}\right\}\right\},
\end{aligned}
$$


Table 1

Dynamics of bird-rat-cat model

\begin{tabular}{lll}
\hline & Existence & Locally asymptotically stability \\
\hline$E_{0}$ & no condition & unstable (i.e., saddle-node) \\
$E_{b}$ & no condition & $d_{r}>\frac{b_{1} K+b_{2}}{1+\delta K}, \quad d_{c}>\frac{c_{1} K}{1+\alpha K}$ \\
& & $0<d_{r}<b_{2}\left(1-\frac{r \sigma}{a_{1}}\right)$, \\
$E_{r}$ & $b_{2}>d r$ & $d_{c}>\frac{c_{2}\left(b_{2}-d_{r}\right)}{\sigma d_{r}+\beta\left(b_{2}-d_{r}\right)}$ \\
& $d_{r}<b_{2}, \quad c_{2} \gamma \geq b_{3} \beta, \quad$ & $0<d_{r}<\min \left\{b_{2}, \frac{a_{1} b_{3}}{\sigma a_{2}}\right\}-\frac{r b_{3}}{a_{2}}$ \\
$E_{r c}$ & $0<d_{c}<\frac{c_{2}\left(b_{2}-d_{r}\right)}{\sigma d_{r}+\beta\left(b_{2}-d_{r}\right)}$ & $c_{1} \gamma \geq \alpha a_{2}, \quad, \quad b_{r}>\max \left\{b_{2}-\frac{b_{3} r}{a_{2}}, \frac{K a_{2} b_{1}+a_{2} b_{2}-b_{3} r}{K a_{2} \delta+a_{2}}\right\}$ \\
$E_{b c}$ & $0<d_{c}<\frac{c_{1} K}{1+\alpha K}$ & $a_{1} \delta \leq b_{1} \sigma$, \\
& $b_{2}<d_{r}<\frac{b_{1} K+b_{2}}{1+\delta K}$ or $\quad b_{1}>\delta b_{2}, \quad d_{c} \geq \max \left\{\frac{c_{1}}{\alpha}, \frac{c_{2}}{\beta}\right\}$ \\
$E_{b r}$ & $0<b_{2}\left(1-\frac{r \sigma}{a_{1}}\right)<d_{r}<\min \left\{b_{2}, \frac{b_{1}}{\delta}\right\}$ &
\end{tabular}

$$
\begin{aligned}
\Delta_{r} & =\left\{\left(d_{r}, d_{c}\right): 0<d_{r}<b_{2}\left(1-\frac{r \sigma}{a_{1}}\right), \quad d_{c}>\frac{c_{2}\left(b_{2}-d_{r}\right)}{\sigma d_{r}+\beta\left(b_{2}-d_{r}\right)}\right\}, \\
\Delta_{r c} & =\left\{\left(d_{r}, d_{c}\right): c_{2} \gamma \geq b_{3} \beta, 0<d_{r}<\min \left\{b_{2}, \frac{a_{1} b_{3}}{\sigma a_{2}}-\frac{r b_{3}}{a_{2}}\right\},\right. \\
0 & \left.<d_{c}<\frac{c_{2}\left(b_{2}-d_{r}\right)}{\sigma d_{r}+\beta\left(b_{2}-d_{r}\right)}\right\} .
\end{aligned}
$$

For (2.2), $E_{i}$ is an attractor in $\Delta_{i}$, where $i=b, b c, b r, r, r c$. Fig. 3.2 illustrates the $d_{r}, d_{c}$ parameter ranges for various extinction and coexistence scenarios with other parameters fixed. It is clear that $\Delta_{r}$ and $\Delta_{r c}$ in Fig. 3.2 are dangerous regions for the birds since the bird component of the attractor of (2.2) is zero. In other words, extinction in these regions is inevitable for the birds.

In view of the above analysis and Fig. 3.2, we see that our criteria for the stability of steady states have room for improvement.

\section{Control of alien species}

The harm caused by introduced species on oceanic islands is widely known, and control programs are recognized as the best way to restore ecosystems (Atkinson, 1988). However, the optimal strategy is not simple to find. In this section, we investigate the effects of various controls of the alien predator species for model (2.2).

As in Courchamp et al. (1999), we apply control efforts on the rat population by varying the artificially imposed death rate $\mu_{r}$ and control efforts on the cat population by varying 
(a) parameters scenarios



(c) rat bifurcation surface

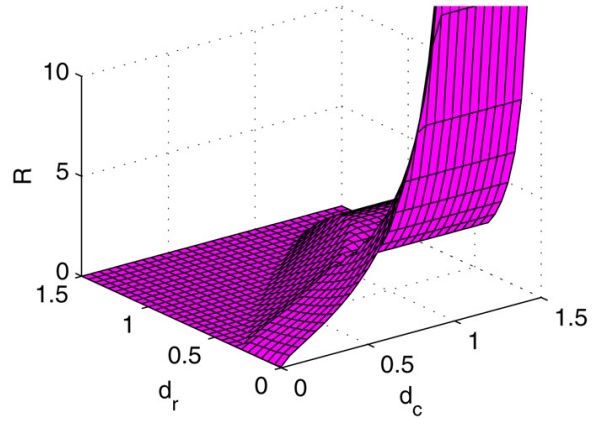

(b) bird bifurcation surface

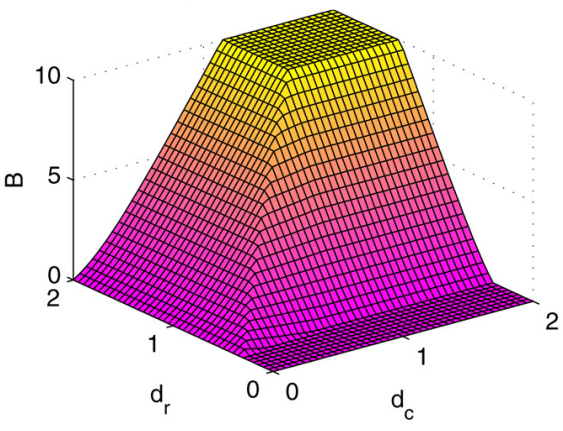

(d) cat bifurcation surface

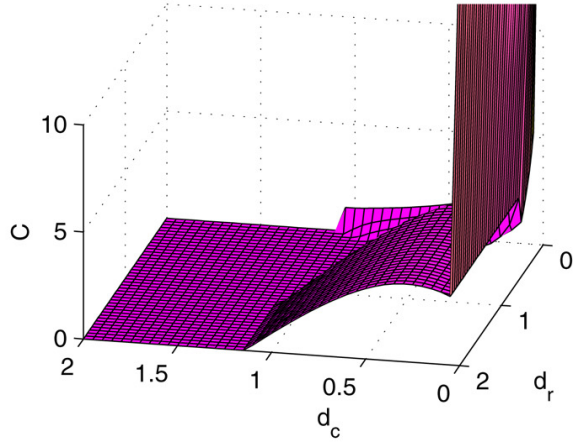

Fig. 3.2. Here $r=0.8, K=10, a_{1}=0.8, a_{2}=0.9, \delta=0.5, \sigma=0.6, \alpha=0.6, \beta=0.5, \gamma=0.8, b 1=0.7$, $b_{2}=1, b_{3}=0.8, c_{1}=0.8, c_{2}=0.55$ and let $d_{r}$ and $d_{c}$ be parameters. (a) Parameter ranges for extinction scenarios and possible coexistence. (b)-(d) Bifurcation diagrams for the bird, the rat and the cat, respectively, with $d_{r}$ and $d_{c}$ being the parameters. In the rest of the $d_{r}-d_{c}$ plane, i.e., $\Delta_{b r c}+$ ?, the bird, the rat and the cat can possibly coexist at a positive equilibrium or coexist cyclicly.

the artificially imposed death rate $\mu_{c}$. The controlled bird-rat-cat system takes the form of

$$
\begin{aligned}
\frac{\mathrm{d} B}{\mathrm{~d} t} & =r B\left(1-\frac{B}{K}\right)-\frac{a_{1} B R}{1+\delta B+\sigma R}-\frac{a_{2} B C}{1+\alpha B+\beta R+\gamma C}, \\
\frac{\mathrm{d} R}{\mathrm{~d} t} & =\frac{b_{1} B R+b_{2} R}{1+\delta B+\sigma R}-d_{r} R-\frac{b_{3} R C}{1+\alpha B+\beta R+\gamma C}-\mu_{r} R, \\
\frac{\mathrm{d} C}{\mathrm{~d} t} & =-d_{c} C+\frac{c_{1} B C+c_{2} R C}{1+\alpha B+\beta R+\gamma C}-\mu_{c} C .
\end{aligned}
$$

When we incorporate the control effort of the rat and the cat into the natural death rate of the rat and the cat, respectively, (4.1) and (2.2) are the same. The qualitative analysis of the dynamics of (4.1) is exactly the same as those in Section 3 except replacing $d_{r}$ and $d_{c}$ with $d_{r}+\mu_{r}$ and $d_{c}+\mu_{c}$ respectively, so the details are omitted here.

In the following discussion, Fig. 3.2 is helpful for us to understand the control strategy of the rat or the cat in order to protect the bird. 


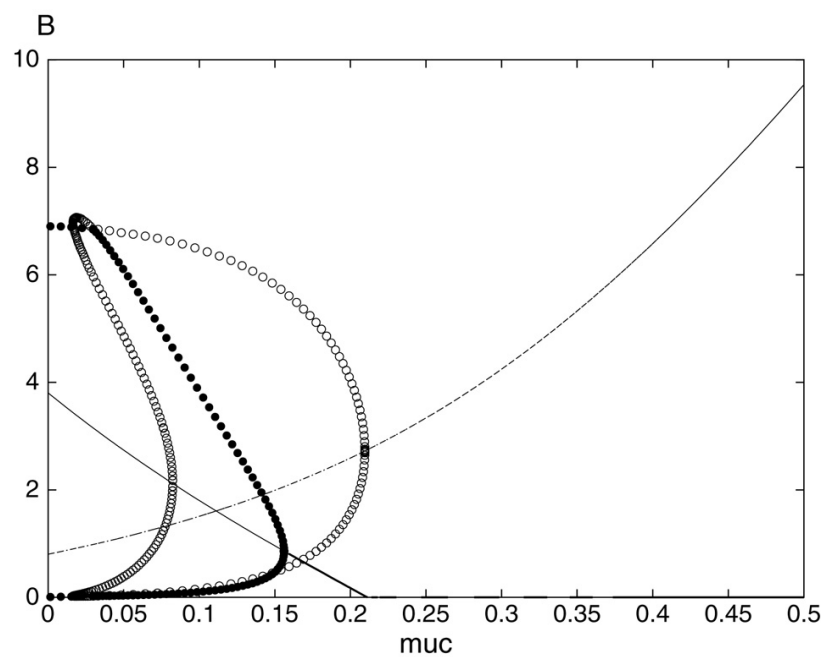

Fig. 3.3. A bifurcation diagram generated by AUTO, using the parameter values in Fig. 4.4(c). See also Fig. 4.6.

\subsection{Protect the bird population by controlling the rat or the cat}

First, we consider the case when the birds are in danger:

$$
0<d_{r}<b_{2}\left(1-\frac{r \sigma}{a_{1}}\right), \quad d_{c}>\frac{c_{2}\left(b_{2}-d_{r}\right)}{\sigma d_{r}+\beta\left(b_{2}-d_{r}\right)} .
$$

In this case, $E_{r}$ is an attractor. The rats have a low death rate while the cats have a high death rate. The cats grow slowly. Consequently, the cats cannot effectively suppress the rats. The rats have a rapid growth and exert a high predation pressure on the birds. $E_{r}$ is the attractor of (4.1). The birds are thus in a very dangerous situation. Theorem 3.2 tells us that if we do not control the rats, the birds are doomed (see, e.g., Fig. 4.1(a)). In order to protect the birds, a effective control program shall be implemented. One can observe that the control or the eradication of the cats cannot save or restore the birds since any increased control effort $\mu_{c}$ of the cats cannot alter the fact that $E_{r}$ is an attractor of (4.1) (see Fig. 4.2(f)). The optimal and effective control strategy for protecting the birds is to control the rats as much as possible. If the control is sufficient, for example, if $d_{r}+\mu_{r}>\left(b_{1} K+b_{2}\right) /(1+\delta K)$ and $d_{c} \mu_{c}>\frac{c_{1} K}{1+\alpha K}$, the rats will be eventually eradicated from the ecosystem and the bird population tends to its environmental carrying capacity (see Fig. 4.1(d) and (f)); if the control is insufficient, i.e., $d_{r}+\mu_{r}<b_{2}\left(1-r \sigma / a_{1}\right)$, birds will die out (i.e., $E_{r}=\left(0,\left(b_{2}-d_{r}-\mu_{r}\right) /\left(\sigma\left(d_{r}+\mu_{r}\right)\right)\right.$, 0$)$ is still the attractor of (4.1)) (see Fig. 4.1(b) and (f)); if the control is mild such that $b_{2}<d_{r}+\mu_{r}<$ $\left(b_{1} K+b_{2}\right) /(1+\delta K)$, then birds and rats will coexist and $E_{b r}$ is an attractor now (see Fig. 4.1(c) and (f)). For this particular set of parameters, the extinction fate of the cats cannot be altered by any control effort on the rats since the cats have a death rate too high. 
(a) $\mu_{\mathrm{r}}=0, \mu_{\mathrm{c}}=0$

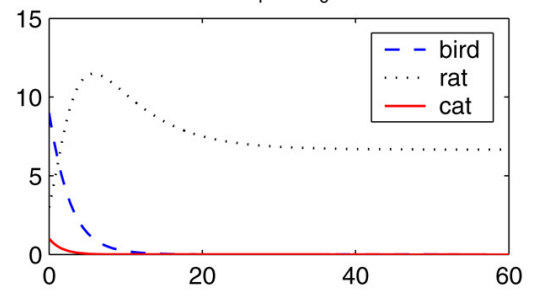

(c) $\mu_{\mathrm{r}}=0.4, \mu_{\mathrm{c}}=0$

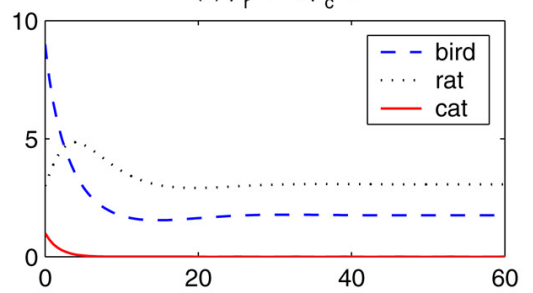

(e) $\mu_{\mathrm{r}}=1.5, \mu_{\mathrm{c}}=0$

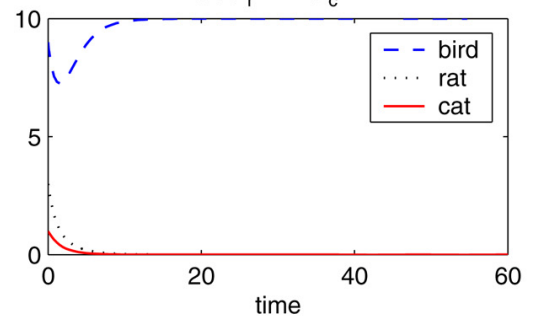

(b) $\mu_{\mathrm{r}}=0.1, \mu_{\mathrm{c}}=0$

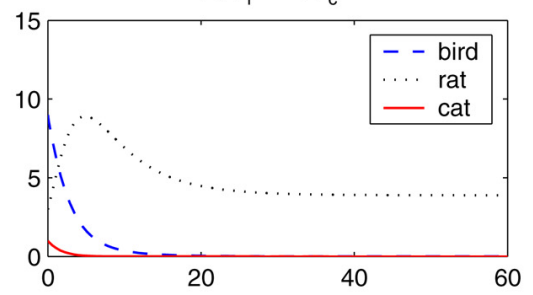

(d) $\mu_{\mathrm{r}}=1, \mu_{\mathrm{c}}=0$

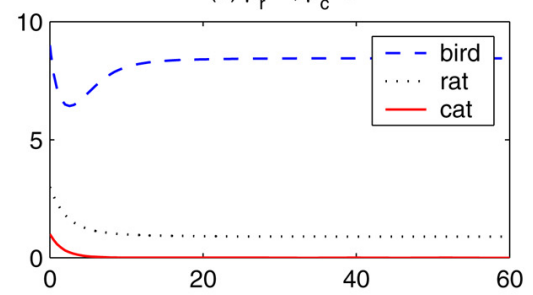

(f) parameters scenario and control strategies

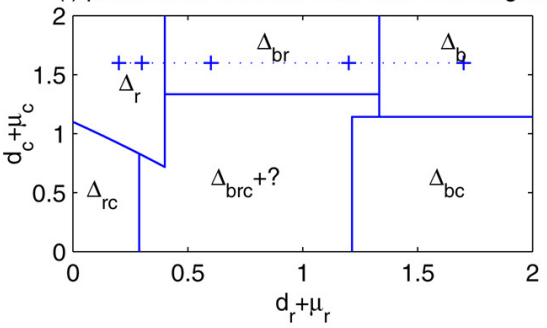

Fig. 4.1. Restore the birds by controlling the rats. (a) Both the birds and the cats will become extinct when there is no control on the rats. (b) Insufficient control of the rats cannot alter the fate of the birds. (c), (d) Sufficient control results in the coexistence of the birds and the rats. (d) With the eradication of the rats, the bird population tends to its carrying capacity. The fate of the cats is doomed regardless of the rat control efforts. The parameters in the above numerical simulations are $r=0.8, K=10, a_{1}=0.8, a_{2}=0.9, \delta=0.5, \sigma=0.6, \alpha=0.6$, $\beta=0.5, \gamma=0.8, b 1=0.7, b_{2}=1, b_{3}=0.8, c_{1}=0.8, c_{2}=0.55, d_{r}=0.2, d_{c}=1.6$.

Now, we investigate the following dangerous situation for the birds:

$$
\begin{aligned}
c_{2} \gamma & \geq b_{3} \beta, \quad 0<d_{r}<\min \left\{b_{2}, \frac{a_{1} b_{3}}{\sigma a_{2}}\right\}-\frac{r b_{3}}{a_{2}}, \\
0 & <d_{c}<\frac{c_{2}\left(b_{2}-d_{r}\right)}{\sigma d_{r}+\beta\left(b_{2}-d_{r}\right)} .
\end{aligned}
$$

In this case, both the rats and the cats have a low death rate, and the cats have to spend more time on interspecies interference than on searching and handling the caught rats (i.e., $c_{2} \gamma \geq b_{3} \beta$ ). In this case, the birds undergo predation pressure from both the rats and the cats. Theorem 3.3 tells us that $E_{r c}$ is an attractor of (2.2), which means that the birds will become extinct and the rats and the cats coexist (see Figs. 4.2 and 4.3(a) and (f)). In order to protect the birds, we have several control strategies: control the cats, control the rats, or control both the cats and the rats. 
(a) $\mu_{\mathrm{r}}=0, \mu_{\mathrm{c}}=0$

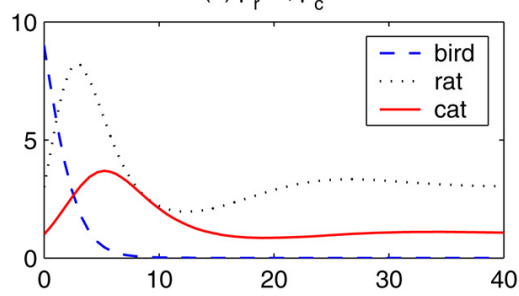

(c) $\mu_{\mathrm{r}}=0, \mu_{\mathrm{c}}=1.2$

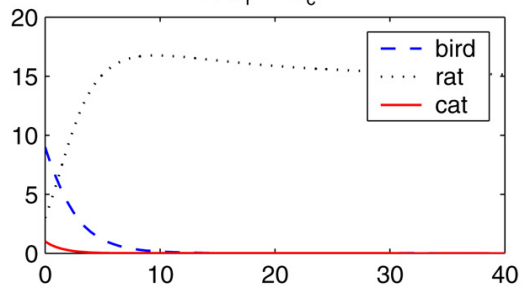

(e) $\mu_{\mathrm{r}}=1.4, \mu_{\mathrm{c}}=1.2$

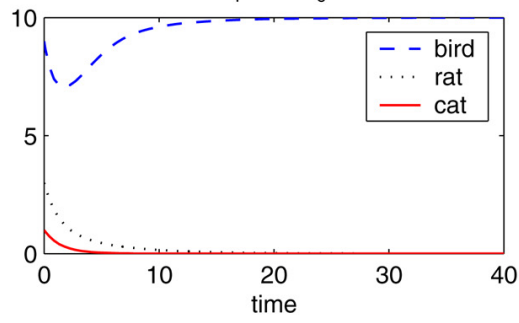

(b) $\mu_{\mathrm{r}}=0, \mu_{\mathrm{c}}=0.7$

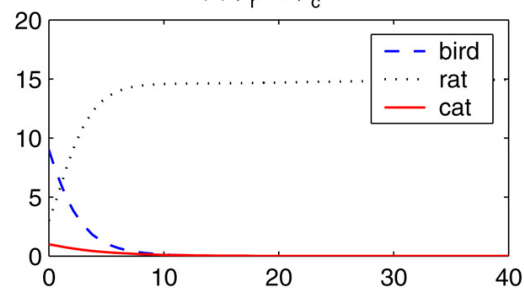

(d) $\mu_{\mathrm{r}}=0.7, \mu_{\mathrm{c}}=1.2$

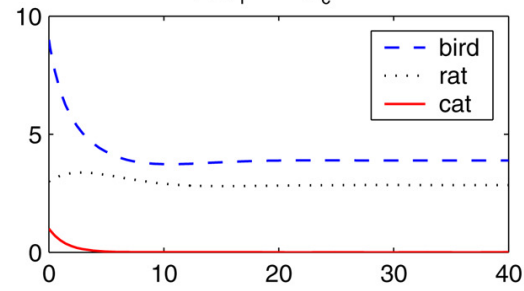

(f) parameters scenario and control strategies

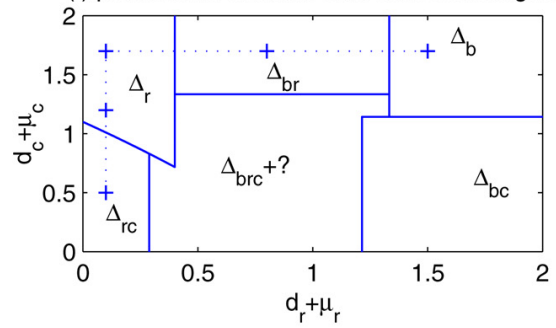

Fig. 4.2. Restore the birds by controlling the cats first and then the rats. (a), (b) Control the cats only. After the control, the cats are eradicated but the control is useless to restore the birds. (d), (e) Control both the cats and the rats. The bird is restored even to its carrying capacity. The parameters used for numerical simulations are same as those in Fig. 3.2 except that $d_{r}=0.1, d_{c}=0.5$.

First, we discuss the control of the cats. If we increase the 'death rate' $d_{c}+\mu_{c}$, then, since the rats have low death rate, one can observe that $E_{r}$ will be the attractor of the system. That is to say, the control of the cats has little effect in protecting the birds. In order to protect the birds, along with a eradicative control of the cats, i.e., $d_{c}+\mu_{c}>\max \left\{c_{1} / \alpha, c_{2} / \beta\right\}$, an effective control of rats must be carried out simultaneously. Mild control of the rats, i.e., $b_{2}<d_{r}+\mu_{r}<\left(b_{1} K+b_{2}\right) /(1+\delta K)$, may result in the coexistence of the birds and the rats (see Fig. 4.2(d) and (f)). If the control of the rats is strong, say, $d_{r}+\mu_{r}>\left(b_{1} K+b_{2}\right) /(1+\delta K)$, then the rats are eliminated from the ecosystem and the bird tends to its carrying capacity (see Fig. 4.2(e) and (f)).

We now consider the control of the rats. After mild control of the rats, the system results in the coexistence of the birds, the rats and the cats, i.e., $E_{b r c}$ is an attractor (see Fig. 4.3(b), (c) and (f)). If the control effort is sufficient enough, say $d_{r}+\mu_{r}>\max \left\{b_{2}, b_{1} / \delta\right\}$, then the rats are eradicated from the system and the birds and the cats coexist at $E_{b c}$ (see Fig. 4.3(d) and (f)). After the eradication of the rat, the control of the cat is more beneficial to the bird. 
(a) $\mu_{\mathrm{r}}=0, \mu_{\mathrm{c}}=0$

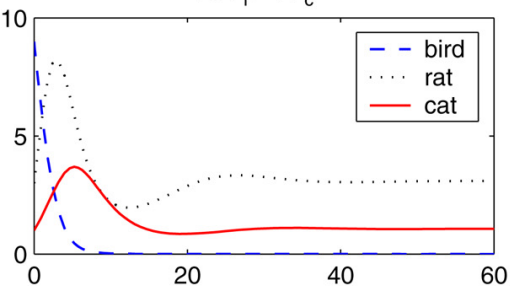

(c) $\mu_{\mathrm{r}}=0.6, \mu_{\mathrm{c}}=0$

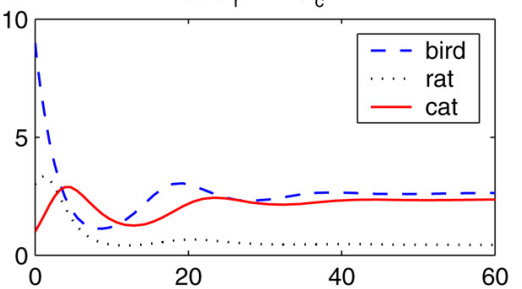

(e) $\mu_{\mathrm{r}}=1.3, \mu_{\mathrm{c}}=0.9$

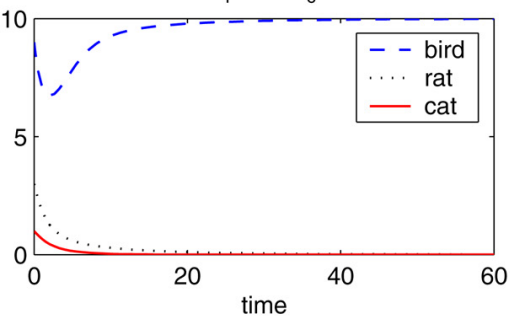

(b) $\mu_{\mathrm{r}}=0.4, \mu_{\mathrm{c}}=0$

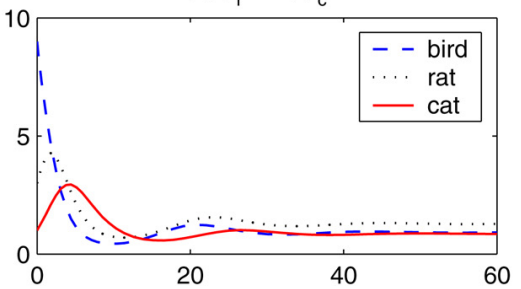

(d) $\mu_{\mathrm{r}}=1.3, \mu_{\mathrm{c}}=0$

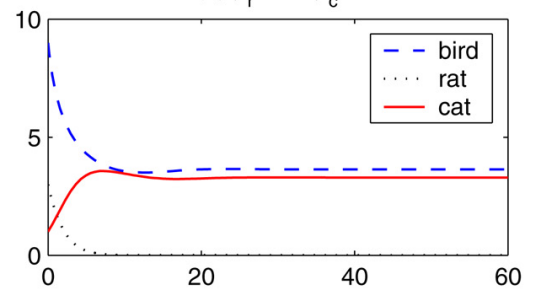

(f) parameters scenario and control strategies

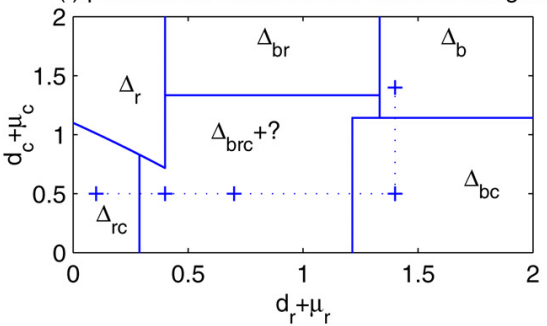

Fig. 4.3. Restore the birds by controlling the rats first and then the cats. (a) No control of the rats or the cats; the birds are in a dangerous situation: the birds become extinct and the rats and the cats coexist. (b), (c) Mediate sufficient control of the rats and no control of the cats: the birds, rats and cats coexist. (d) Sufficient control of the rats and not control of the cats: the rats are eradicated and the birds and the cats coexist. (e) Following the control of the rats, the eradication control results in the fact that the bird reaches its carrying capacity. The parameters used for numerical simulations are same as those in Fig. 4.2.

Sufficient control of the cat can help the bird increase toward its carrying capacity (see Fig. 4.3(e) and (f)).

\subsection{Mesopredator release effect}

Although in some cases, the control of cat has been proved to be effective in restoring some endangered ecosystems, such strategies are not universally applicable. In some cases, it may cause a disastrous impact to managed or natural ecosystems. The mesopredator release effect is widely known to ecologists, and it is one typical impact among the results of eradication efforts of a superpredator. For the convenience of discussion, we define two types of mesopredator release effects: severe mesopredator release, where once superpredators (cats) are suppressed, a burst of mesopredators (rats) follows which drives their shared prey (birds) to extinction, and mild mesopredator release, where the 
(a) $\mu_{c}=0.05$

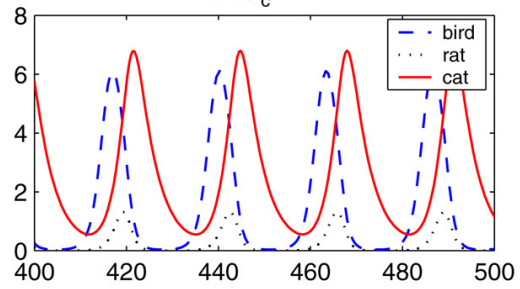

(c) bifurcation curve: bird vs $\mu_{c}$

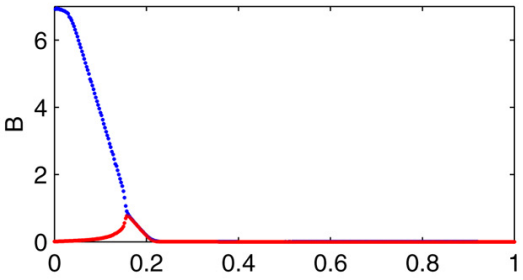

(e) $\mu_{r}=0, \mu_{c}=0.5$

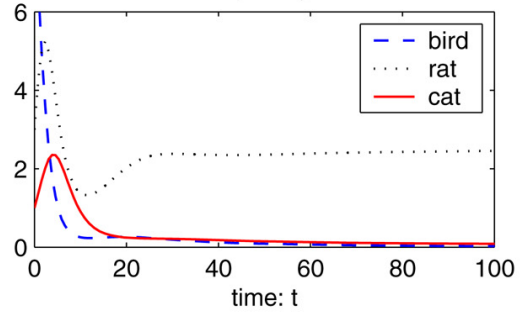

(b) $\mu_{c}=0.1$

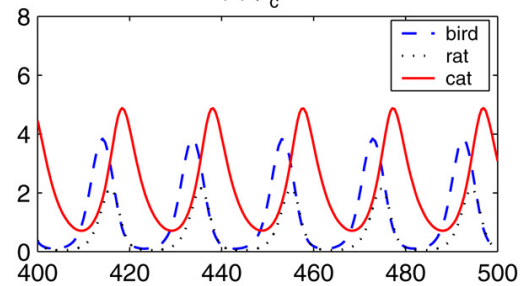

(d) $\mu_{r}=0, \mu_{c}=0$

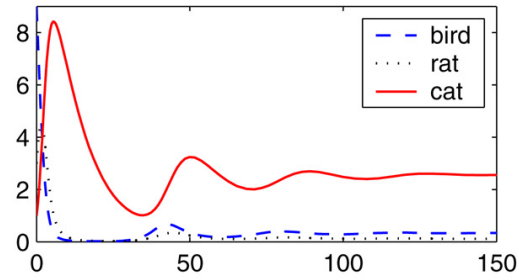

(f) bifurcation curve: bird vs $\mu_{c}$

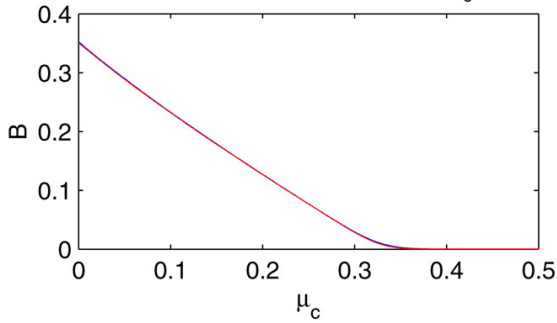

Fig. 4.4. Severe mesopredator release effect. (a)-(c) $r=1, K=10, a_{1}=0.8, a_{2}=0.7, \delta=0.5, \sigma=0.5$, $\alpha=0.6, \beta=0.4, \gamma=0.2, b_{1}=0.6, b_{2}=1, b_{3}=0.7, d_{c}=0.2, c_{1}=0.5, c_{2}=0.5, d_{r}=0.15, d_{c}=0.2$, $\mu_{r}=0.1$. (d)-(f) the parameters are same as those in Fig. 3.2 except that $d_{r}=0.38, d_{c}=0.1$.

mesopredator release could assert negative impact on the endemic prey (birds) but does not cause the extinction of the endemic prey.

In order to locate and understand the conditions for the occurrence of mesopredator release, let us start with some examples of (4.1), where the bird, the rat and the cat coexist cyclicly or at an equilibrium (see Fig. 4.4(a) and (d)). In order to eradicate the cat from the ecosystem, we increase the control effort $\mu_{c}$ from 0 to $\mu=0.2$ and then to $\mu=0.5$. This will eradicate the cats successfully (see Fig. 4.4(b) and (c), (e) and (f)). After the eradication of the cats, the birds are also eradicated by the rats as a result of expansion of the rat population resulting from their predators being removed. That is to say, we have theoretically proved the existence of the so-called mesopredator release effect.

We claim:

Severe mesopredator release occurs if $d_{r}<b_{2}\left(1-r \sigma / a_{1}\right)$.

In fact, if $d_{r}<b_{2}\left(1-r \sigma / a_{1}\right)$, the final destiny of (2.2) is a trichotomy: the birds die out and the rats and the cats coexist; or the birds, the rats and the cats coexist at an equilibrium or cyclicly; or the rats survive and the birds and the cats die out. Although the birds, 
(a) $\mu_{\mathrm{r}}=0, \mu_{\mathrm{c}}=0$

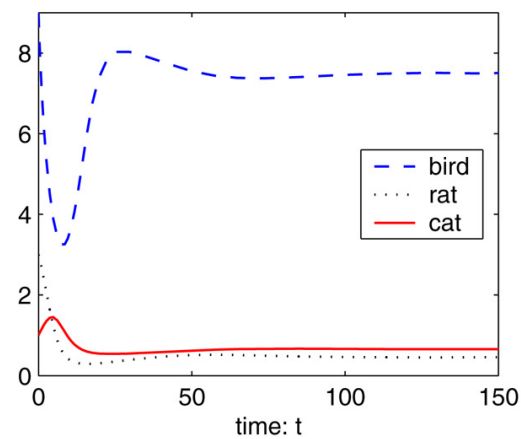

(c) $\mu_{\mathrm{r}}=0, \mu_{\mathrm{c}}=0.5$

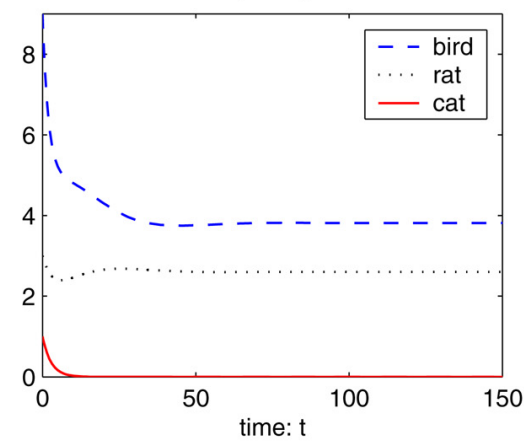

(b) $\mu_{\mathrm{r}}=0, \mu_{\mathrm{c}}=0.1$

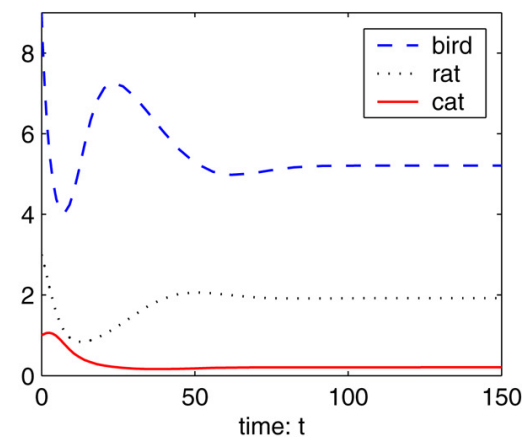

(d) bifurcation diagram for bird

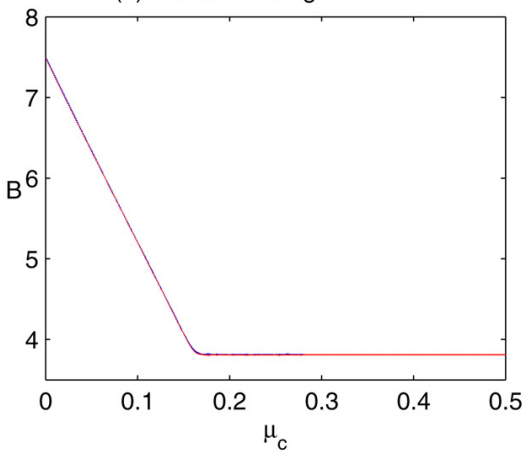

Fig. 4.5. Mild mesopredator release effect. (a) Bird, rat and cat coexist at an equilibrium. (b), (c) Increase the control effort $\mu_{c}$, then the cat are successfully eradicated. Although the eradication of cats does not lead to the extinction of birds, the rats keep the bird population size at a lower level. $\left(r=0.5, K=10, a_{1}=0.5, a_{2}=0.7\right.$, $\delta=0.5, \sigma=0.5, \alpha=0.6, \beta=0.4, \gamma=0.2, b_{1}=0.2, b_{2}=0.5, b_{3}=0.9, c_{1}=0.5, c_{2}=0.7, d_{r}=0.3$, $\left.d_{c}=0.7\right)$

the rats and the cats can coexist, the bird population density is usually very small and the birds remain endangered. So, the control strategy to save the birds must be carried out. The control or eradication of the cats is among the choices. If one adopts the control strategy to eradicate the cats, when $d_{c}+\mu_{c}>c_{2}\left(d_{2}-d_{r}\right) /\left(\sigma d_{r}+\beta\left(b_{2}-d_{r}\right)\right)$, Theorem 3.2 tells us that $E_{r}$ will be the attractor, that is to say, the birds die out following the eradication of the cat. Severe mesopredator release occurs.

On the other hand, if $d_{r}>b_{2}\left(1-r \sigma / a_{1}\right)$, from the theorems in Section 3 and Fig. 3.2, one can easily observe that, under any control strategies, especially the control or eradication of the cat, the bird will not become extinct although it can be at a lower level. That is to say, in this case, severe mesopredator release cannot occur.

The control or eradication of the cat does not always trigger severe mesopredator release. In some cases, moderate mesopredator release does occur (see for example Fig. 4.5). Although the mesopredator release does not lead to the extinction of the bird, the control or eradication of the cat is still detrimental to the bird since, after the eradication of 
(a) bifurcation surface

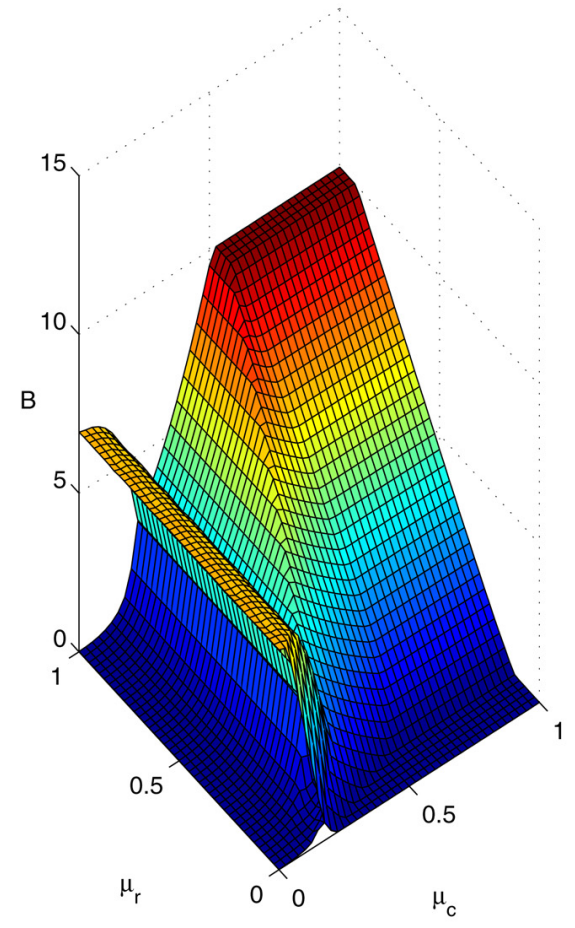

(b) bifurcation curve with $\mu_{r}=0.85$

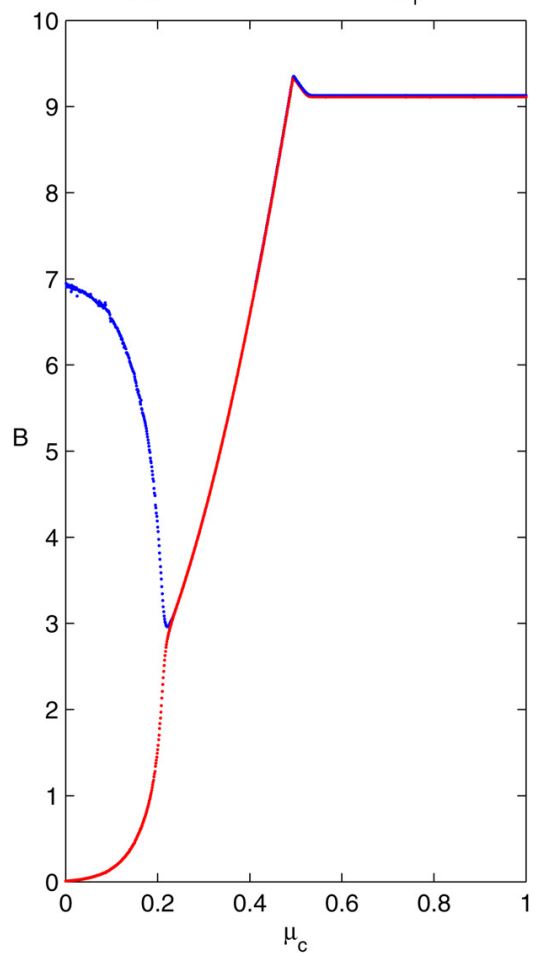

Fig. 4.6. This figure shows that the control of the cat can trigger a mild mesopredator release, and, in some cases, it is positive to the bird. The parameters are the same as those in Fig. 4.4(c).

the cat, the rat put the bird species in a relatively lower level. However, in some other cases, the control or eradication of the cat is beneficial to the bird (see, e.g., Figs. 3.2 and 4.6).

\section{Discussion}

In this paper, we have developed a plausible model (2.2) describing the dynamics of a three-species prey-mesopredator-superpredator interaction. Our model overcomes several model formulation problems arising in system (1.1) in Courchamp et al. (1999). We show that (2.2) can admit richer and more realistic dynamics than that of system (1.1). Therefore it provides much more information for conservation biology, especially for the design of control programs. Based on (2.2), our study of the control strategies shows that the fate of the prey sensitively depends on both the superpredator control level and the mesopredator control level.

When $d_{r}<b_{2}\left(1-r \sigma / a_{1}\right)$, our findings strongly suggest that superpredator control or eradication should be avoided since it will trigger a severe mesopredator release, which leads to the extinction of the endemic prey (bird). This statement provides a set of reasonable answers to the questions $\mathrm{A}$ and $\mathrm{B}$ posted in the introduction section. In the case of 
$d_{r}<b_{2}\left(1-r \sigma / a_{1}\right)$, the optimal control strategy is to control the rats as soon and as much as possible, and the control effort $\mu_{r}$ must be greater than $b_{2}\left(1-r \sigma / a_{1}\right)-d_{r}$. Otherwise, the control strategy cannot save the bird. After the sufficient and effective control of the rat, one can control the cat further since the control of the cat is now beneficial to the bird.

When $d_{r}>b_{2}\left(1-r \sigma / a_{1}\right)$, the bird will persist although the bird population size can possibly be very small. Our suggestion is to control the rats first, then control the cats, or to control the rats and the cats simultaneously.

In real applications, if the ecosystem on some island can be well described by the prey-mesopredator-superpredator trophic web and the indigenous prey species is endangered, in order to protect the endemic prey species, we shall carry out some well conceived control strategy. First, we should determine the status of the ecosystem, i.e., determine the parameters in (2.2), then study the qualitative dynamics of the system. It is a good idea to compute some diagrams similar to Fig. 3.2 and determine the positions of the system in them. Then design the optimal control strategies based on the above discussion, policy restrictions, financial constrains and so on.

Our simulation work shed some light on the following interesting question: assuming that in some ecosystems rats are present and are capable of driving the birds to extinction, can one introduce a superpredator (e.g., cats) to ensure meaningful biological control? This study suggests that the answer can be positive since the removal of cats may have negative aspects on birds. However, their introduction cannot be lightly recommended in most circumstances since real ecosystems are very complicated and species are often intricately linked.

A yet to be addressed mathematical question on (2.2) is under what conditions all three species coexist. This is the so-called persistence question. Global stability of the positive equilibrium will ensure this, but it is much more difficult to work on. In this paper, we do not study this topic since the persistence issue is not directly relevant to biological control applications and the mesopredator release effect. An even more intriguing mathematical question is whether (2.2) is capable of generating chaotic dynamics. Our intensive but also highly selective computational efforts so far fail to produce that.

\section{Acknowledgements}

We would like to thank the referees for their valuable suggestions that enabled us to improve the presentation of this paper. The first author was supported by the National Natural Science Foundation of PR China (No. 10171010 and 10201005), the key project on science and technology of the education ministry of the People's Republic of China (No. Key 01061) and the Science Foundation of Jilin Province of PR China for distinguished young scholars. The second author was partially supported by NSF grants DMS-0077790 and DMS-0342388. The third author was partially supported by NSF grant DMS-0314575 and JMSF grant 220020052.

\section{References}

Apps, P.J., 1986. A case study of an alien predator (Felis catus) introduced on Dassen Island: selective advantages. South African Antarctic Research 16, 118-122. 
Ariditi, R., Perrin, N., Saiah, H., 1991. Functional responses and heterogeneities: an experimental test with cladocerans. Oikos 60, 69-75.

Arnaud, G., Rodriguez, A., Ortega-Rubio, A., Alvarez-Cardenas, S., 1993. Predation by cats on the unique endemic lizard of Socorro Island Urosaurus auriculatus Revillagigedo Mexico. Ohio Journal of Science 93, 101-104.

Atkinson, I.A.E., 1988. Opportunities for ecological restoration. New Zealand Journal of Ecology 11, 1-12.

Atkinson, I.A.E., 1989. Introduced animals and extinctions.. In: Western, D., Pearl, M.C. (Eds.), Conservation for the Twenty-first Century. Oxford University Press, New York, pp. 54-75.

Beddington, J.R., 1975. Mutual interference between parasites or predators and its effect on searching efficiency. Journal of Animal Ecology 44, 331-340.

Bloomer, J.P., Bester, M.N., 1990. Diet of declining feral cat Felis catus population on Marion Island. South African Journal of Wildlife Research 20, 1-4.

Case, T.J., Bolger, D.T., 1991. The role of introduced species in shaping the distribution and abundance of island reptiles. Evolutionary Ecology 5, 272-290.

Courchamp, F., Langlais, M., Sugihara, G., 1999. Cats protecting birds: modelling the mesopredator release effect. Journal of Animal Ecology 68, 282-292.

Crooks, K.R., Soule, M.E., 1999. Mesopredator release and avifaunal extinctions in a fragmented system. Nature $400,563-566$.

Diamond, J., 1989. Overview of recent extinctions. In: Western, D., Pearl, M.C. (Eds.), Conservation for the Twenty-first Century. Oxford University Press, New York, pp. 37-41.

Dobson, A.P., 1988. Restoring island ecosystems: the potential of parasite to control introduced mammals. Conservation Biology 2, 31-39.

Dolman, P.M., 1995. The intensity of interference varies with resource density: evidence from a field study with snow buntings, Plectrophenax nivalis. Oecologia 102, 511-514.

Domm, S., Messersmith, J., 1990. Feral cat eradication on a barrier reef island Australia. Atoll Research Bulletin $338,1-4$.

Fan, M., Kuang, Y., 2004. Dynamics of a nonautonomous predator prey system with the Beddington-DeAngelis functional response. Journal of Mathematical Analysis and Applications 295, 15-39.

Fitzgerald, B.M., 1988. Diet of domestic cats and their impact on prey populations. In: Turner, D.C., Bateson, P. (Eds.), The Domestic Cat, the Biology of its Behaviour. Cambridge University Press, pp. 123-147.

Fitzgerald, B.M., Turner, D.C., 2000. Hunting behaviour of domestic cats and their impact on prey populations. In: Turner, D.C., Bateson, P. (Eds.), The Domestic Cat, the Biology of its Behaviour. Cambridge University Press, pp. 151-175.

Holling, C.S., 1965. The functional response of predators to prey density and its role in mimicry and population regulation. Memoirs of the Entomological Society of Canada 45, 5-60.

Hwang, T.W., 2003. Global analysis of the predator-prey system with Beddington-DeAngelis functional response. Journal of Mathematical Analysis and Applications 281, 395-401.

Hwang, T.W., 2004. Uniqueness of limit cycles of the predator-prey system with Beddington-DeAngelis functional response. Journal of Mathematical Analysis and Applications 290, 113-122.

Hwang, T.W., Kuang, Y., 2003. Deterministic extinction effect of parasites on host populations. Journal of Mathematical Biology 46, 17-30.

Iverson, J.B., 1978. The impact of feral cats and dogs on populations of the west Indian rock iguana, Cyclura carinata. Biological Conservation 14, 63-73.

Jost, C., 1998. Comparing predator-prey models qualitatively and quantitatively with ecological time-series data, Ph.D. Thesis, Institut National Agronomique, Paris-Grignon, France.

Jost, C., Ellner, S.P., 2000. Testing for predator dependence in predator-prey dynamics: a non-parametric approach. Proceedings of the Royal Society of London Series B 267, 1611-1620.

King, W.B., 1985. Island birds: will the future repeat the past? In: Moors, P.J. (Ed.), Conservation of Island Birds, ICBP Technical Publication, no. 3, Cambridge, pp. 3-15.

Liberg, O., Sandell, M., Pontier, D., Natoli, E., 2000. Density, spatial organisation and reproductive tactics in the domestic cat and other felids. In: Turner, D.C., Bateson, P. (Eds.), The Domestic Cat, the Biology of its Behaviour. Cambridge University Press, pp. 119-147.

Mellink, E., 1992. The Status of Neotoma anthonyi Rodentia Muridae Cricetinae of Todos Santos Islands Baja California Mexico. Bulletin Southern California Academy of Sciences 91, 137-140. 
Mooney, H.A., Hobbs, R.J., 2000. Invasive Species in a Changing World. Island Press.

Moors, P.J., Atkinson, I.A.E., 1984. Predation on seabirds by introduced animals, and factors affecting its severity. In: Croxall, J.P., Evans, P.G.H., Schreiber, R.W. (Eds.), Status and Conservation of the World's Seabirds, ICBP Technical Publication, No. 2, Cambridge, pp. 667-690.

Murphy, E., Bradfield, P., 1992. Change in diet of stoats following poisoning of rats in a New-Zealand forest. New Zealand Journal of Ecology 16, 137-140.

Nogales, M., Medina, F.M., 1996. A review of the diet of feral domestic cats (Felis silvetris L catus) on the Canary Islands with new data from the laurel forest of La Gomera. Z. Säugetierkunde 61, 1-6.

Nogales, M., Rodriguez, J.L., Delgado, G., Quilis, V., Trujillo, O., 1992. The diet of feral cats (Felis catus) on Alegranza island (north of Lanzarote, Canary Islands). Folia Zoologica 41, 209-212.

Rauzon, M.J., 1985. Feral cats on Jarvis Island: their effects and their eradication. Atoll Research Bulletin 282, $1-30$.

Rodriguez-Estrella, R., Arnaud, G., Cardenas, S.A., Rodriguez, A., 1991. Predation by feral cats on birds at Isla Socorro Mexico. Western Birds 22, 141-143.

Rogers, C.M., Caro, M.J., 1998. Song sparrows, top carnivores, and nest predation: a test of the mesopredator release hypothesis. Oecologia 116, 227-233.

Rogers, C.M., Heard, S.B., 2000. The mesopredator release hypothesis: integrating landbird management with ecological theory. Studies in Avian Biology 21, 138-143.

Russo, E., 2004. Cooperation urged on invasives, The Scientist, March 22, 2004.

Skalski, G.T., Gilliam, J.F., 2001. Functional responses with predator interference: viable alternatives to the Holling type II model. Ecology 82, 3083-3092.

Smith, F.E., 1963. Population dynamics in Daphnia Magna and a new model for population growth. Ecology 44, 651-663.

Soule, M.E., Bolger, D.T., Alberts, A.C., Wright, J., Sorice, M., Hill, S., 1988. Reconstructed dynamics of rapid extinctions of chaparral-requiring birds in urban habitat islands. Conservation Biology 2, 75-92.

Spencer, P.B.S., 1991. Evidence of predation by a feral cat, Felis catus (Carnivora: Felidae) on an isolated rockwall-aby colony in tropical Queensland. Australian Mammalogy 14, 143-144.

Stapp, P., 2002. Stable isotopes reveal evidence of predation by ship rats on seabirds on the Shiant Islands, Scotland. Journal of Applied Ecology 39, 831-840.

Taylor, R.H., Thomas, B.W., 1993. Rats eradicated from rugged Breaksea island 170 ha Fiordland New Zealand. Biological Conservation 65, 191-198.

Towns, D.R., 1996. Changes in habitat use by lizards on a New Zealand island following removal of the introduced Pacific Rat Rattus exulans. Pacific Conservation Biology 2, 286-292.

Williamson, M., 1996. Biological Invasion. Chapman \& Hall.

Zavaleta, E., Hobbs, R.J., Mooney, H.A., 2001. Viewing invasive species removal in a whole-ecosystem context. TREE 16, 454-459. 